\title{
Quark number fluctuations at high temperatures
}

\author{
Peter Petreczky ${ }^{a}$ Prasad Hegde $^{b}$ and Alexander Velytsky ${ }^{*, a}$ (RBC-Bielefeld \\ Collaboration) \\ ${ }^{a}$ Department of Physics, Brookhaven National Laboratories, Upton, NY11733, USA. \\ E-mail: petreczk@quark.phy.bnl.gov \\ E-mail: velequark.phy.bnl.gov \\ ${ }^{b}$ Dept. of Physics and Astronomy, SUNY Stony Brook, Stony Brook, NY11790, USA. \\ E-mail: phegde@quark.phy.bnl.gov
}

\begin{abstract}
We calculate the second, fourth and sixth order quark number fluctuations in the deconfined phase of 2+1 flavor QCD using lattices with temporal extent $N_{t}=4,6,8$ and 12. We consider light, strange and charm quarks. We use $\mathrm{p} 4$ action for valence quarks and gauge configurations generated with $\mathrm{p} 4$ action with physical value of the strange quark mass and light quark mass $m_{q}=0.1 m_{s}$ generated by the RBC-Bielefeld collaboration. We observe that for all quark masses the quark number fluctuations rapidly get close to the corresponding ideal gas limits. We compare our results to predictions of a quasi-particle model and resummed high temperature perturbative calculations. We also investigate correlations among different flavor channels.
\end{abstract}

The XXVII International Symposium on Lattice Field Theory

July 26-31, 2009

Peking University, Beijing, China

* Speaker. 


\section{Introduction}

Quark number fluctuations are basic observables which can be obtained by taking derivatives of the grand canonical potential with respect to quark chemical potentials $\mu_{q}$. Here we look directly at the related derivatives $c_{i}^{q}$, which enter into the Taylor expansion of the pressure at finite chemical potentials $\left(\mu_{q} / T \lesssim 1\right)$

$$
\frac{p}{T^{4}}=\frac{1}{V T^{3}} \ln Z\left(V, T, \mu_{f}\right)=\left.\frac{p}{T^{4}}\right|_{\mu=0}+\sum_{i, j, k, l} c_{i, j, k, l}^{u, d, s, c}(T)\left(\frac{\mu_{u}}{T}\right)^{i}\left(\frac{\mu_{d}}{T}\right)^{j}\left(\frac{\mu_{s}}{T}\right)^{k}\left(\frac{\mu_{c}}{T}\right)^{l} .
$$

Their combinations form fluctuations of conserved charges (see ref. [1] for a related lattice study). Thus they can be used to study the properties of a thermal medium produced in heavy ion collisions [2]. From Eq. (1.1) it is clear that the expansion coefficients are related to the quark number densities $n_{f}$, quark number susceptibilities $\chi_{2}^{f} \equiv\left\langle n_{f}^{2}\right\rangle=2 c_{2}^{f}$ and other moments: $\chi_{4}^{f} \equiv\left\langle n_{f}^{4}\right\rangle-3\left\langle n_{f}^{2}\right\rangle^{2}=24 c_{4}^{f}, \ldots$

In this work we are interested in the behavior of the quark number fluctuations at high temperatures $240 \mathrm{MeV}<T<900 \mathrm{MeV}$ and zero chemical potential. We would like to study the approach of relevant observables to the ideal gas (Stefan-Boltzmann (SB) limit) [3]

$$
\frac{p_{S B}}{T^{4}}=\Omega^{(0)}(T, \mu)=\frac{8 \pi^{2}}{45}+\sum_{f=u, d, . .}\left[\frac{7 \pi^{2}}{60}+\frac{1}{2}\left(\frac{\mu_{f}}{T}\right)^{2}+\frac{1}{4 \pi^{2}}\left(\frac{\mu_{f}}{T}\right)^{4}\right] .
$$

We use the tree level Symanzik improved gauge action and improved staggered fermion $\mathrm{p} 4-$ action with 3-link smearing (p4fat3). The gauge configurations were generated for calculation of the equation of state with $(2+1)$ flavors using almost physical light quark masses resulting in $m_{\pi} \approx 220 \mathrm{MeV}$ and physical $s$ quark mass [ 4 ]. In our measurements we add non-dynamical $c$ quark. In table 1 we list the gauge configurations used in the study.

\begin{tabular}{c|c|c|c|c||c|c|c|c|c} 
lattice & $\beta$ & $T$ & configurations & Rnd & lattice & $\beta$ & $T$ & configurations & Rnd \\
\hline $32^{3} \times 8$ & 4.08 & 539.19 & $24100^{*}$ & 96 & $32^{3} \times 4$ & 3.92 & 861 & 6500 & 96 \\
$32^{3} \times 8$ & 4.00 & 474.84 & $23200^{*}$ & 96 & $16^{3} \times 4$ & 3.82 & 706 & 10000 & 96 \\
$32^{3} \times 8$ & 3.92 & 416.42 & $27100^{*}$ & 96 & $16^{3} \times 4$ & 3.76 & 622 & 11650 & 96 \\
$32^{3} \times 8$ & 3.82 & 350.66 & $15100^{*}$ & 96 & $16^{3} \times 4$ & 3.69 & 532 & 9450 & 96 \\
$32^{3} \times 8$ & 3.76 & 314.53 & 6050 & 96 & $16^{3} \times 4$ & 3.63 & 465 & 10000 & 96 \\
$32^{3} \times 8$ & 3.63 & 242.78 & 6000 & 96 & $16^{3} \times 4$ & 3.57 & 404 & 21150 & 96 \\
& & & & & $16^{3} \times 4$ & 3.54 & 373 & 6250 & 96 \\
& & & & & $16^{3} \times 4$ & 3.51 & 347 & 10000 & 96 \\
& & & & & $16^{3} \times 4$ & 3.49 & 330 & 9400 & 96 \\
\hline $32^{3} \times 6$ & 4.08 & 745 & 6800 & 96 & $32^{3} \times 12$ & 3.76 & 212 & 3150 & 100 \\
$32^{3} \times 6$ & 4.00 & 633 & $10000^{*}$ & 96 & $32^{3} \times 12$ & 3.82 & 240 & 3750 & 100 \\
$32^{3} \times 6$ & 3.92 & 553 & 9300 & 96 & $32^{3} \times 12$ & 3.92 & 273 & 4000 & 100 \\
$32^{3} \times 6$ & 3.82 & 451 & 8400 & 96 & & & & & \\
\hline
\end{tabular}

Table 1: Lattice parameters and the number of trajectories for each parameter set. The step size between measurements is 100 trajectories for stared entries $\left(^{*}\right)$ and 50 for the rest. Typically we start measurements after 1000 trajectories. Rnd is the number of random vectors used in the stochastic estimators. 


\section{Numerical results}

Here we present our numerical results for quark number fluctuations up to the 6th order, including second order flavor off-diagonal fluctuations. In Fig. 1 we plot the off-diagonal coefficients $c_{11}^{u d}$ and $c_{11}^{u s}$ normalized by the related diagonal quadratic terms $c_{2}^{u}$ and $c_{2}^{s}$. The plot indicates that the correlations between $u$ and $d$, and $u$ and $s$ flavors vanish rapidly after the deconfinement. This is suggestive of the fact that there are no bound states in the deconfinement phase [5]. Similar
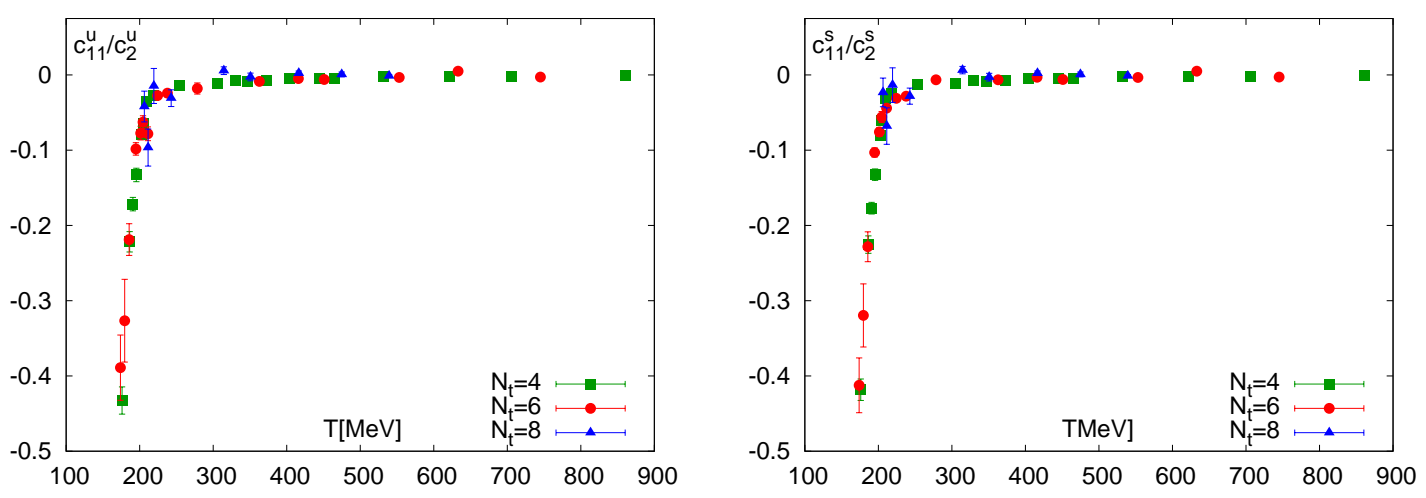

Figure 1: The off-diagonal coefficient $c_{11}^{u d}$ (left) and $c_{11}^{u s}$ (right) normalized by $c_{2}^{u}$ and $c_{2}^{s}$ respectively.

results were obtained for $u c$ correlations. Thus we may expect that quark gas description should be reasonable for quark number fluctuations.
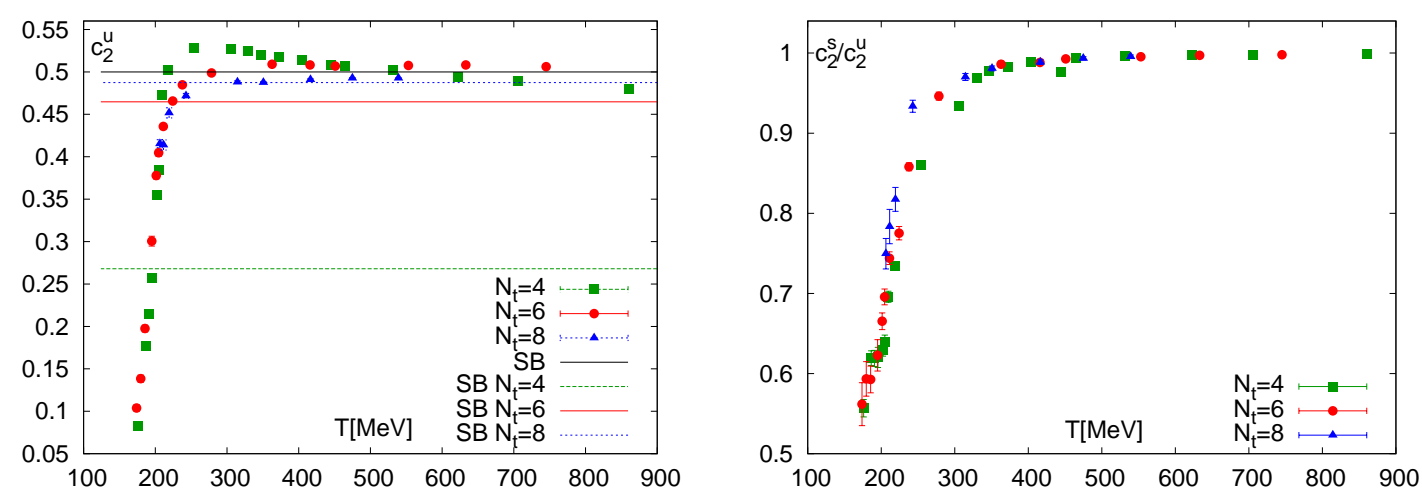

Figure 2: Numerical results for $c_{2}^{u}$ calculated for different $N_{t}$ and the corresponding SB values (left). The ratio $c_{2}^{s} / c_{2}^{u}$ as function of the temperature (right).

In the left panel of Fig. 2 we present our lattice results for $c_{2}^{u}$ coefficients as well as the corresponding continuum SB limit. In addition we plot the SB value corrected for a finite lattice cutoff. In Fig. 2 we also show our previous (low temperature) results from Ref. [1]. We see that the observed cutoff effects are different and smaller than the cutoff effects in the free theory. The difference between the $N_{t}=6$ and $N_{t}=8$ results is about $10 \%$. Therefore to establish the continuum limit calculations on $N_{t}=12$ lattices are needed. Similar results were obtained for $c_{2}^{s}$. 
The right panel of Fig. 2 shows the ratio $c_{2}^{s} / c_{2}^{u}$. As this has been discussed in [1, 6] this ratio approaches unity at $T \geq 300 \mathrm{MeV}$. This in fact is expected in the quark gas picture.

In the left panel of Fig. 3 we compare our $c_{2}^{u}$ results for $T \geq 200 \mathrm{MeV}$ with the resummed high temperature perturbative calculations [7]. This figure clearly shows the $10 \%$ difference between $N_{t}=6$ and $N_{t}=8$ results. Therefore we also did calculations at $N_{t}=12$ lattices at 3 temperatures. The corresponding results are also shown in the figure. At the highest temperature, where $N_{t}=12$ data are available, we see a reasonably good agreement with $N_{t}=8$ data. This suggests that $N_{t}=8$ calculations are sufficiently close to the continuum results. Therefore the comparison of $N_{t}=8$ data with the resummed perturbative calculations performed in the continuum limit is meaningful and shows that quark number fluctuations are well described by the resummed perturbative calculations.
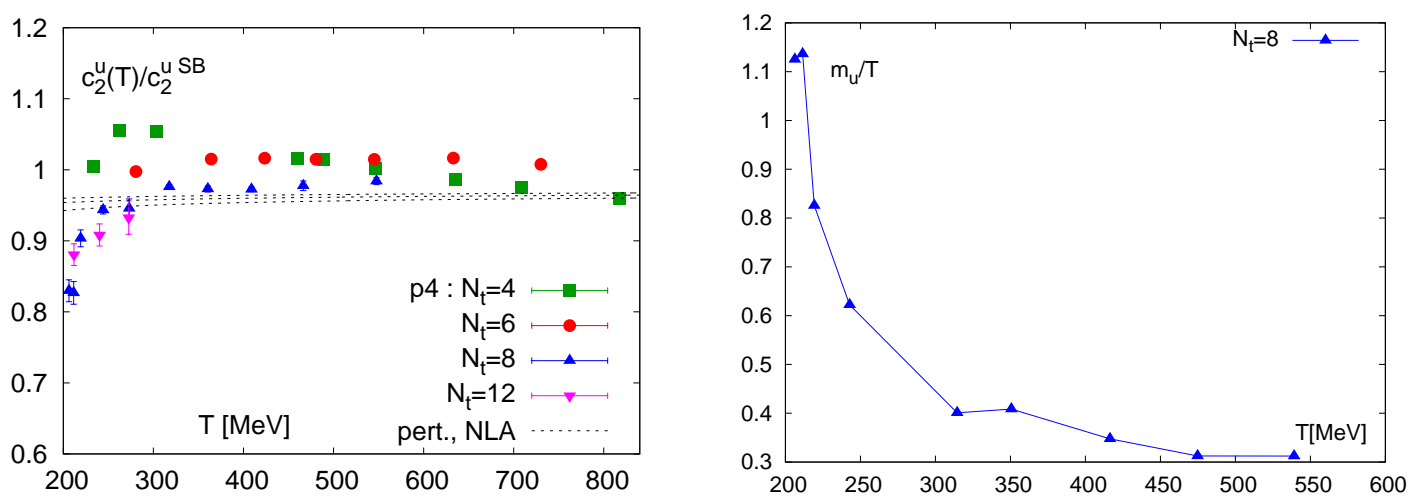

Figure 3: Left panel: The ratio of $c_{2}^{u}$ to the corresponding SB value compared to the high temperature perturbative results shown as dashed lines. The three lines correspond to the renormalization scale $\mu=$ $\pi T, 2 \pi T$ and $4 \pi T$ (from bottom to top). Right panel: The effective thermal quark mass extracted from $c_{2}^{u}$.

We also calculated the 4th order expansion coefficients $c_{4}^{u}$ and $c_{4}^{s}$ in the high temperature regime. The numerical results for $c_{4}^{u}$ are shown in Fig. $\bigoplus$ (the results for $c_{4}^{s}$ are similar at high temperature). In the figure we show the corresponding SB value in the continuum as well as for finite $N_{t}$. The cutoff dependence of $c_{4}$ is similar to that of $c_{2}$. Namely, the observed cutoff effects are different from the free theory. Also the ordering of $N_{t}=6$ and $N_{t}=8$ results is reversed. The difference between them is less than $10 \%$. Assuming that $N_{t}=8$ results are close to the continuum limit, as the $c_{2}$ calculations suggest, we see deviations from the ideal gas limit around $20 \%$, i.e. two times more than for $c_{2}$.

In the resummation scheme used in [7] the dominant contribution to the quark number fluctuations is given by quasi-particles with effective quark mass equal to the hard thermal loop mass. Therefore it makes sense to compare the lattice results to a simple massive quasi-particle model. The pressure for massive quark gas is given by equation (2.1) [B]

$$
\begin{aligned}
\frac{p_{m}}{T^{4}} & =\frac{6}{\pi^{2}}\left(\frac{m}{T}\right)^{2} \sum_{l=1}^{\infty}(-1)^{l+1} l^{-2} K_{2}(l m / T) \cosh \left(l \mu_{q} / T\right) \\
c_{2} & =\frac{1}{2} \frac{6}{\pi^{2}}\left(\frac{m}{T}\right)^{2} \sum_{l=1}^{\infty}(-1)^{l+1} K_{2}(l m / T) .
\end{aligned}
$$




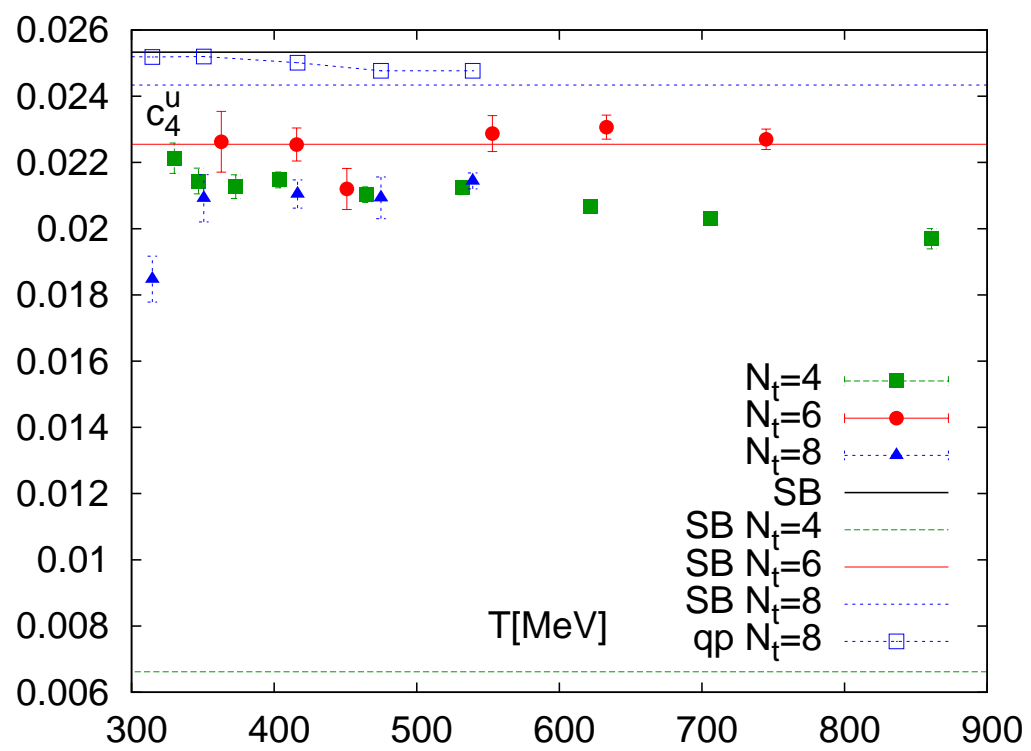

Figure 4: The high temperature behavior of $c_{4}^{u}$.
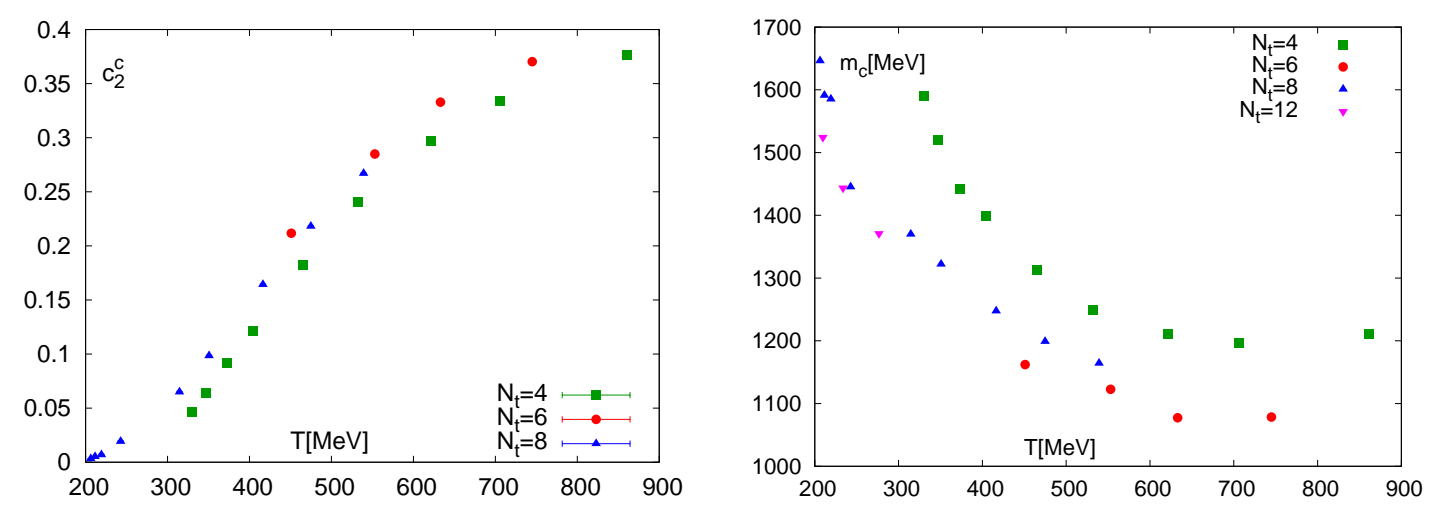

Figure 5: Numerical results for $c_{2}^{c}$ (left) and the effective mass extracted from it (right).

Taking successive derivatives of the rhs with respect to $\mu / T$ we obtain expressions for $c_{2}$ (2.2), $c_{4}$, etc. The expression for $c_{2}$ can be solved for $m / T$ using $c_{2}$ values obtained numerically. The corresponding quasi-particle masses are shown in Fig. B (right) for $N_{t}=8$. We can use these effective masses to make predictions for $c_{4}$ and $c_{6}$. The quasi-particle results for $c_{4}^{u}$ is shown in Fig. 4. As we can see they are close to the massless ideal gas limit and almost $20 \%$ off from the numerical data for $N_{t}=8$. This indicates that the simplest quasi-particle description is not adequate for light quarks. Unfortunately the are no perturbative calculations for $c_{4}^{u}$. It would be interesting to see whether the resummed perturbative calculations similar to those performed for $c_{2}^{u}$ in [7] could explain the observed deviation from the ideal gas limit for $c_{4}^{u}$.

Now we consider the charm quark case. In Fig. 5 on the left panel we plot the results for $c_{2}^{c}$. Here one can notice that $N_{t}=4$ data shows slightly different scaling due to cutoff effects. On the 
right panel we present the effective mass extracted from the $c_{2}^{c}$ values at each temperature point. In Fig. 6 we compare $c_{4}^{c}$ data with the quasi-particle model results, where the effective mass was

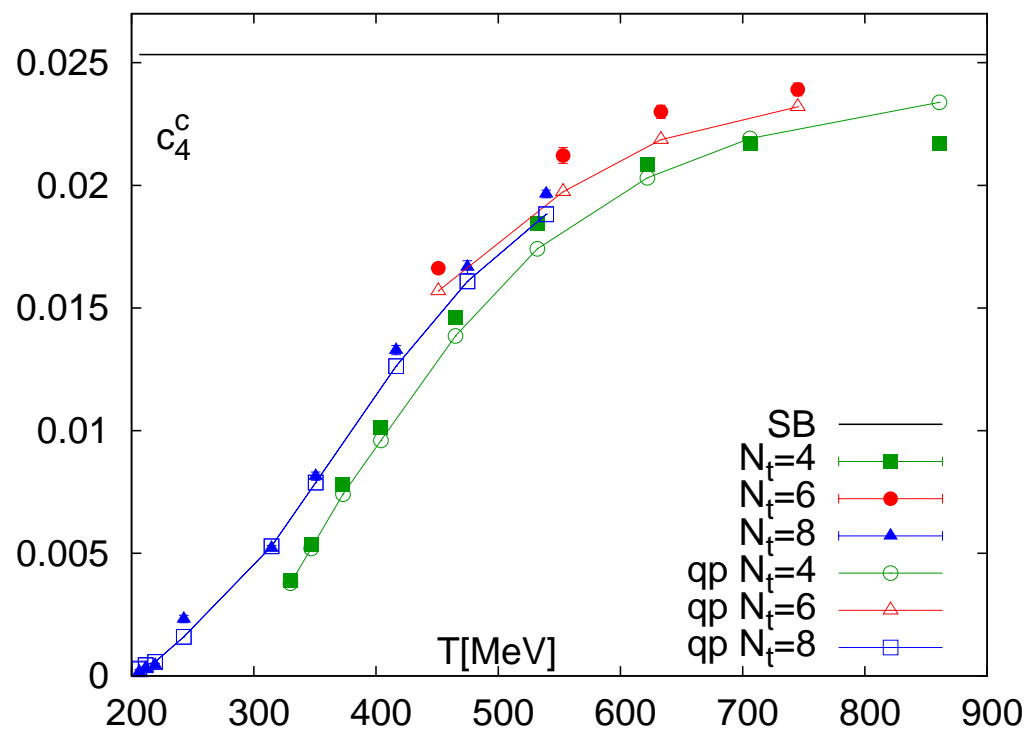

Figure 6: Numerical results for $c_{4}^{c}$ and the quasi-particle results.

computed from $c_{2}^{c}$ data. We see a very good agreement between the quasi-particle model results and the numerical data. This indicates that for heavy charm quark the quasi-particle description is adequate for modeling of $c_{4}^{c}$ at high temperature.

As we see from Figs. 5 and 6 the fluctuations of the charm quark number is comparable to those of the light quark sector at the highest temperatures. This means that at temperatures $T \sim 800 \mathrm{MeV}$ the contribution of charm quark to QCD thermodynamics is significant as this has been suggested in Ref. [9] using weak coupling analysis.

We also considered the 6th order coefficient. In Fig. 7 we present the results for $c_{6}^{u}$ and $c_{6}^{c}$. The light quark result according to Eq. (1.2) should be zero for a massless ideal gas. The numerical result agrees reasonably with this prediction. For the charm quark number fluctuation $c_{6}^{c}$ we took the effective mass computed from $c_{2}^{c}$ and used it to compute the massive ideal gas estimate, which we plot in the figure with lines for various $N_{t}$. It is interesting that the numerical results for all $N_{t}$ are in reasonable agreement with the quasi-particle model results in the deconfined regime.

\section{Conclusions}

In this contribution we discussed quark number fluctuations $c_{2}, c_{4}$ and $c_{6}$ calculated with $\mathrm{p} 4$ action on $N_{t}=4,6,8$ and 12 lattices. We see that the cutoff dependence of quark number fluctuations is different from the expectation based on the free theory. Off-diagonal fluctuations vanish soon after the deconfinement transition. This suggests that fluctuations can be understood in terms of the quark gas. In the light quark sector the quadratic fluctuations are quite close to the massless 


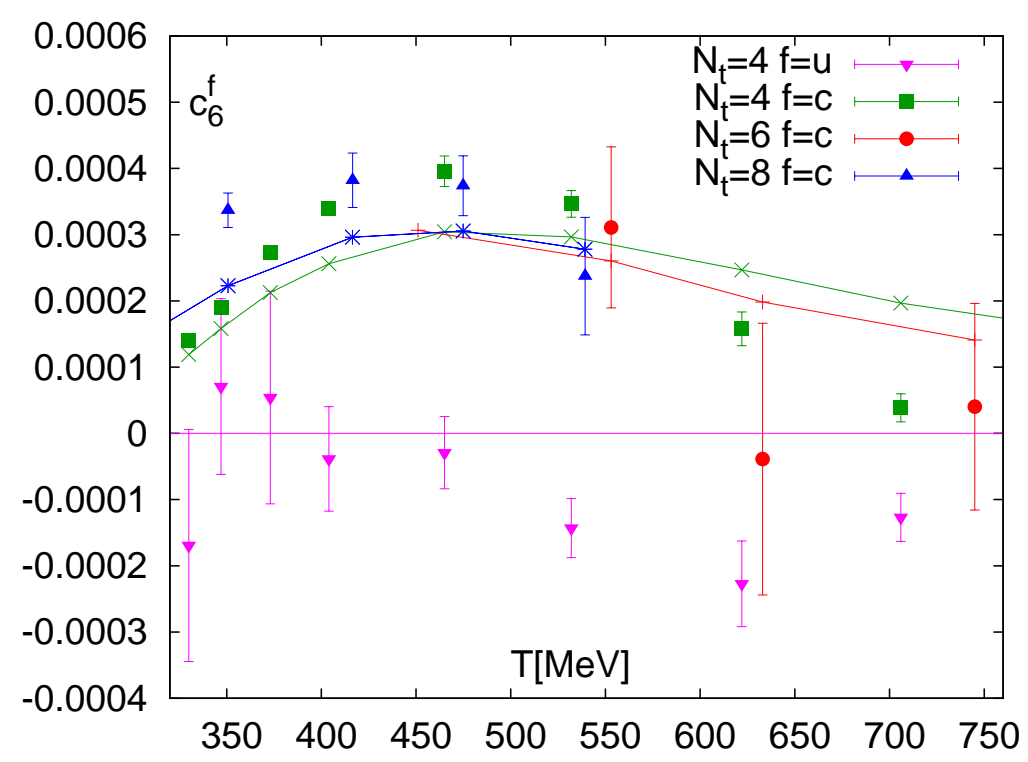

Figure 7: The sixth order $c_{6}$ results for $c$ and $u$ flavors.

ideal gas limit and agree with the resummed perturbative calculations and expectations of the quasiparticle model. The quartic fluctuations on the other hand show larger (about 20\%) deviation from the massless ideal gas limit and do not agree with the predictions of the quasi-particle model. In the charm quark sector we find that all fluctuations up to 6th order can be reasonably well described by the quasi-particle model. Current results suggest that continuum limit may be reached for $N_{t}=8$ but calculations on $N_{t}=12$ lattices at higher temperature are needed to verify this statement.

\section{Acknowledgments}

This work was supported by U.S. Department of Energy under Contract No. DE-AC0298CH10886.

\section{References}

[1] M. Cheng et al., Phys. Rev. D 79, 074505 (2009) [arXiv:0811.1006 [hep-lat]].

[2] V. Koch, arXiv:0810.2520 [nucl-th].

[3] C. R. Allton et al., Phys. Rev. D 71, 054508 (2005) [arXiv:hep-lat/0501030].

[4] M. Cheng et al., Phys. Rev. D 77, 014511 (2008) [arXiv:0710.0354 [hep-lat]].

[5] V. Koch, A. Majumder and J. Randrup, Phys. Rev. Lett. 95, 182301 (2005) [arXiv:nucl-th/0505052].

[6] A. Bazavov et al., Phys. Rev. D 80, 014504 (2009) [arXiv:0903.4379 [hep-lat]].

[7] A. Rebhan, arXiv:hep-ph/0301130.

[8] F. Karsch, K. Redlich and A. Tawfik, Phys. Lett. B 571, 67 (2003) [arXiv:hep-ph/0306208].

[9] M. Laine and Y. Schroder, Phys. Rev. D 73, 085009 (2006) [arXiv:hep-ph/0603048]. 